OPEN ACCESS

Edited by:

Carl E. Stafstrom, University of WisconsinMadison, USA

Reviewed by:

Luca Bartolini,

National Institute of Neurological

Disorders and Stroke, USA

Emily Camm,

University of Cambridge, UK

${ }^{*}$ Correspondence:

Young-Mock Lee

ymleemd@yuhs.ac

Specialty section: This article was submitted to Neuropediatrics, a section of the journal Frontiers in Neurology

Received: 19 January 2017 Accepted: 27 April 2017

Published: 17 May 2017

Citation:

Eom S and Lee Y-M (2017) Long-term Developmental Trends of Pediatric Mitochondrial Diseases: The

Five Stages of Developmental

Decline.

Front. Neurol. 8:208

doi: 10.3389/fneur.2017.00208

\section{Long-term Developmental Trends of Pediatric Mitochondrial Diseases: The Five Stages of Developmental Decline}

\author{
Soyong Eom ${ }^{1}$ and Young-Mock Lee ${ }^{2 *}$ \\ ${ }^{1}$ Epilepsy Research Institute, Yonsei University College of Medicine, Seoul, Korea (Republic of), ${ }^{2}$ Department of Pediatrics, \\ Gangnam Severance Hospital, Yonsei University College of Medicine, Seoul, Korea (Republic of)
}

Mitochondrial diseases (MDs) are a heterogeneous group of progressive multisystem disorders caused by impaired mitochondrial function. This study aimed to evaluate the clinical course and long-term development of 53 pediatric patients with MDs. Developmental function was evaluated at nine time points (two pre-diagnosis, one at diagnosis, and six post-diagnosis), with the developmental quotient (DQ) from the Korean infant and child development test (KICDT) assessing a child's developmental age (rather than chronological age). Additionally, disease-related clinical variables were reviewed, and clinical progress was determined through observation. Subgroup analyses by epilepsy severity, syndromic diagnosis, diffuse brain atrophy, and clinical rating were performed. The pre- and post-diagnosis results were compared by the paired $t$-test and Bonferroni correction. The pre-diagnostic, diagnostic, and post-diagnostic evaluations were compared using repeated measures ANOVA. Patients with diffuse brain atrophy at the first pre-diagnostic and second post-diagnostic evaluations showed lower DQs. Compared with patients with a mildly or severely deteriorating clinical course, those with an improving or static clinical course presented higher DQs at the pre-diagnostic and diagnostic evaluations. The age at onset of the first symptom correlated positively with the DQ post-diagnosis. Follow-up revealed consistent patterns of significant developmental deterioration during the lead time to diagnosis, with no significant decline post-diagnosis. The $D Q$ is a feasible predictor and a measure of long-term functional development in children with MD. Early initiation of treatment may minimize developmental regression.

Keywords: mitochondrial disease, developmental quotient, metabolic disease, pediatric epilepsy, brain atrophy

\section{INTRODUCTION}

Mitochondrial diseases (MDs) vary considerably in terms of their manifestations and effects on different organ systems, as well as in terms of age of onset and rate of progression (1). Clinical pediatric reports have described the spectrum of signs and symptoms associated with MDs. Classification relies on accurate clinical, biochemical, and genetic information and may be based on either genotype or phenotype, but there is significant overlap. Although the prognosis for MD is dependent on the underlying diagnosis, there is large phenotypic variation $(2,3)$. Thus, the diagnostic evaluation is necessarily multitiered and broad based, with a focus on integrating information from many avenues (4). In particular, children with encephalopathy experience many limitations in daily 
life (5). However, information on the long-term clinical course and developmental outcome of this heterogeneous group of diseases is lacking (5-7).

One of the key needs of families caring for children with rare metabolic or genetic impairments is to understand the long-term effect of the disorder on the child's development (8). Specifically, a better understanding of the needs, problems, and long-term developmental trends of these children is essential for effective care planning and improving the quality of life (1).

Accordingly, the aims of this study were to evaluate the clinical course and long-term developmental trends in pediatric patients with MDs. The present study is the first to attempt to use a developmental scale for retrospectively assessing and describing the long-term developmental trends of children with MDs.

\section{MATERIALS AND METHODS}

\section{Patients and Procedures}

Data regarding pediatric patients were obtained from hospital records. Patient selection was based on both the diagnosis of $\mathrm{MD}$ and the results of a developmental evaluation using the developmental quotient (DQ). All patients were diagnosed with mitochondrial respiratory chain complex defects by biochemical enzyme assay of muscle tissue samples and satisfied the modified MD criteria proposed by Bernier et al. (9).

Standardized evaluations of developmental function were conducted. In addition, disease-related clinical variables were examined, including age at symptom onset, age at diagnosis, lead time to diagnosis, type of first symptom, lactic acidosis, pathological features indicating myopathies, result of the biochemical enzyme assay for the mitochondrial respiratory chain complex, neuroimaging findings, and syndromic diagnosis.

Lead time to diagnosis was defined as the time between first symptom onset and diagnosis of MD. The severity of serum lactic acidosis was categorized as mild, moderate, or severe, based on the increase over normal reference values ( $\geq 2$-fold, $\geq 3$-fold, or $\geq 4$-fold increase). Normal reference range of lactate is known as $0.5-2.2 \mathrm{mmol} / \mathrm{L}$ (4). Myopathies were classified depending on whether abnormal pathology was detected only on one type of microscopy (one pathology) or on both light and electron microscopies (two pathologies). The diagnoses of mitochondrial encephalomyopathy, lactic acidosis, and stroke-like episodes (MELAS) and Leigh disease were based on the diagnostic criteria reported by Yatsuga et al. (10) and Rahman et al. (11), respectively. Involvement of the central nervous system, either with or without the involvement of other organs, was taken into consideration in assessing the severity of organ involvement in MD. The interpretation of patients' diffuse brain atrophy on MRIs by the radiologist was reviewed, and the results were classified by three levels as no atrophy, mild atrophy, and severe atrophy by the pediatric neurologist.

The patients were divided into three groups based on the presence, severity, and intractability of epilepsy: patients without epilepsy; patients with drug-responsive epilepsy, defined as an epileptic condition responsive to antiepileptic treatment; and patients with drug-resistant epilepsy, requiring more than two tolerated and appropriately chosen and used antiepileptic drug schedules (12). Clinical progress was classified as improving, static, mildly deteriorating, or severely deteriorating, based on the changes in the clinical features of the patient, as observed by the treating clinician (modified from a previously published study) (13).

After the diagnosis of MD was confirmed, all patients were treated with mitochondrial cocktails such as coenzyme Q, carnitine, and various vitamins (14). All procedures conducted were approved by the institutional review board of Gangnam Severance Hospital in Seoul, Korea.

\section{Measures of Developmental Function}

Developmental function was evaluated using the Korean infant and child development test (KICDT). This test was developed by the Development Evaluation Enacting Subcommittee of the Korea Pediatrics Academy in February 2002. The KICDT uses a development index to assess the development of toddlers younger than 5 years of age, whose development level is below the expected based on their chronological age in months. The KICDT can be used to quickly screen toddlers who are suspected of having delayed development or to observe toddlers who are already receiving treatment after positive diagnosis of delayed development (15). The KICDT is a 140 -item, clinician-rated inventory of skills designed to assess a child's developmental age (rather than chronological age) in five functional domains: gross motor, fine motor, social-personal, language, and cognitive-adaptive skills.

The DQ was calculated for each KICDT domain using the following formula: $\mathrm{DQ}=$ (developmental age/chronological age) $\times 100$. The mean of the five domain DQs was designated the overall DQ and was used as the primary index of the child's overall developmental level. An overall DQ below 80 was regarded as abnormal.

The attending pediatricians administered the KICDT and obtained the DQ scores for a total of up to nine evaluations. Pre-diagnostic developmental evaluations were performed twice (the first visit prior to diagnosis, pre-first and the second visit prior to diagnosis, pre-second), followed by a one-time diagnostic evaluation and six post-diagnostic developmental evaluations (the first visit after diagnosis, post-first; the second visit after diagnosis, post-second; the third visit after diagnosis, post-third; the fourth visit after diagnosis, post-fourth; the fifth visit after diagnosis, post-fifth; and the sixth visit after diagnosis, postsixth). To analyze the overall developmental trends, we included the results of the developmental evaluations at all nine time points. For further analyses (patient subgroups and DQ subdomains), only five DQ values at the following time points were used: two pre-diagnostic evaluations (pre-first and pre-second), one-time evaluation during the diagnosis, and two post-diagnostic evaluations (post-first and post-second).

\section{Statistical Analysis}

Univariate (mean, SD, range) and multivariate (Pearson correlation) descriptive statistics were used to analyze patients' characteristics and clinical variables. In addition, Student's $t$-test and the Mann-Whitney $U$-test (for non-parametric ordinal data) were performed to compare the subgroups in terms of epilepsy severity, syndromic diagnosis, diffuse brain atrophy, and clinical 
rating. The paired $t$-test with Bonferroni correction was used to compare the results of the developmental function evaluations (overall DQ and DQ for each KICDT domain) at pre- and postdiagnostic visits for various subgroups of patients and in the total cohort. Repeated measures ANOVA was performed to compare the evaluation results during the pre-diagnostic, diagnostic, and post-diagnostic visits. A post hoc test with Bonferroni adjustment was also conducted. IBM SPSS version 20.0 (IBM, Armonk, NY, USA) was used for data processing and analysis.

\section{RESULTS}

\section{Patient Characteristics}

Our study included 53 children diagnosed with MD, whose developmental function had been assessed at several time points (pre-diagnosis, at diagnosis, and post-diagnosis) between March 2006 and February 2015. The lead time to diagnosis was $1.09 \pm 1.15$ years (range, $0.6-1.46$ years), and the follow-up duration from pre-first to post-second was 5.45 years.

Forty patients $(76 \%)$ had been diagnosed with non-specific MD, 11 (21\%) with Leigh syndrome, and 2 (4\%) with MELAS. The mean age at the first onset of symptoms was $1.0 \pm 1.1$ years (range, $0-4.2$ years), and 32 (60\%) of the children were boys. The most common first symptom was seizure $(26,49 \%)$, followed by delayed development $(22,42 \%)$.

The majority of patients showed static $(20,38 \%)$ or mildly deteriorating $(22,42 \%)$ clinical progress, whereas the rest exhibited severely deteriorating $(10,19 \%)$ or improving $(1,2 \%)$ clinical progress. More details of the clinical characteristics are presented in Tables 1 and 2.

\section{Effects of Syndromic Diagnosis, Drug- Resistant Epilepsy, Diffuse Brain Atrophy, and Clinical Rating}

In patients diagnosed via syndromic diagnosis, no significant differences in DQ were observed between patients with Leigh disease and those with non-specific MD (Table 3). Patients with MELAS were excluded from this analysis because the small number of MELAS cases was insufficient for a meaningful statistical analysis. Similarly, no significant difference in DQ was noted between the subgroups of patients with various types of epilepsy severity (Table 3). However, the presence of diffuse brain atrophy was associated with significantly lower DQ levels of development at the pre-first $(78.6 \pm 20.0$ vs $44.3 \pm 35.3, p=0.047)$ and postsecond evaluations $(33.8 \pm 10.0$ vs $23.7 \pm 13.4, p=0.033)$, with similar but not statistically significant trends for the rest of the evaluations. Furthermore, DQ was significantly higher in patients with improving or static clinical progress than in patients with mildly deteriorating or severely deteriorating clinical progress at pre-second $(68.5 \pm 27.1$ vs $39.70 \pm 29.9, p=0.002)$ and diagnosis $(44.5 \pm 22.8$ vs $25.6 \pm 23.4, p=0.018)$.

\section{Effect of Age at First Symptom Onset and Lead Time to Diagnosis}

Age at the first symptom onset showed positive correlation with the level of developmental function at post-first $(r=0.438$,
TABLE 1 | Diagnostic evaluation of children with MD $(n=53)$.

\begin{tabular}{|c|c|c|}
\hline \multicolumn{2}{|l|}{ Characteristic } & Value \\
\hline \multicolumn{2}{|l|}{ Male sex } & $32(60)$ \\
\hline \multicolumn{2}{|c|}{ Age at first symptom onset, years } & $0.95 \pm 1.07$ \\
\hline \multicolumn{2}{|l|}{ Age at diagnosis, years } & $3.12 \pm 2.49$ \\
\hline \multicolumn{2}{|l|}{ Lead time to diagnosis, years } & $2.17 \pm 2.11$ \\
\hline First symptom & $\begin{array}{l}\text { Seizure } \\
\text { Delayed development } \\
\text { Visual disturbance } \\
\text { Ataxia } \\
\text { Dystonia } \\
\text { Nystagmus } \\
\text { Hearing impairment }\end{array}$ & $\begin{array}{c}26(49) \\
22(42) \\
1(2) \\
1(2) \\
1(2) \\
1(2) \\
1(2)\end{array}$ \\
\hline Lactic acidosis severity & $\begin{array}{l}\text { Normal } \\
\text { Mild } \\
\text { Moderate } \\
\text { Severe }\end{array}$ & $\begin{array}{c}23(43) \\
22(42) \\
7(13) \\
1(2)\end{array}$ \\
\hline $\begin{array}{l}\text { Myopathies by pathologic } \\
\text { features }\end{array}$ & $\begin{array}{l}\text { Normal } \\
\text { One pathology } \\
\text { Two pathologies }\end{array}$ & $\begin{array}{l}10(19) \\
23(43) \\
20(38)\end{array}$ \\
\hline Biochemical enzyme assay & $\begin{array}{l}\text { MRC I complex deficiency } \\
\text { MRC II complex deficiency } \\
\text { MRC IV complex deficiency }\end{array}$ & $\begin{array}{c}48(92) \\
1(2) \\
3(6)\end{array}$ \\
\hline Syndromic diagnosis & $\begin{array}{l}\text { Leigh syndrome } \\
\text { MELAS } \\
\text { Non-specific MD }\end{array}$ & $\begin{array}{c}11(21) \\
2(4) \\
40(76)\end{array}$ \\
\hline
\end{tabular}

Data given as total number (percentage) or mean $\pm S D$.

$M D$, mitochondrial disease; MRC, mitochondrial respiratory chain; MELAS,

mitochondrial encephalopathy, lactic acidosis, and stroke-like episodes.

TABLE 2 | Clinical progress of children with mitochondrial disease $(n=53)$.

\begin{tabular}{llc}
\hline Characteristic & & Value \\
\hline Number of organs involved & & $1.54 \pm 0.93$ \\
Organ involvement & CNS only & $34(64)$ \\
& CNS + other organs & $19(36)$ \\
Severity of epilepsy & No epilepsy & $10(19)$ \\
& Drug-responsive epilepsy & $18(33)$ \\
& Drug-resistant epilepsy & $26(48)$ \\
Diffuse brain atrophy on MRI & Normal & $22(42)$ \\
& Mild & $26(49)$ \\
& Severe & $5(9)$ \\
Clinical progress & Improving & $1(2)$ \\
& Static & $20(38)$ \\
& Mildly deteriorating & $22(42)$ \\
& Severely deteriorating & $10(19)$ \\
\hline
\end{tabular}

Data given as total number (percentage) or mean $\pm S D$.

CNS, central nervous system; MRI, magnetic resonance imaging.

$p=0.047)$ and post-second $(r=0.619, p=0.014)$. Lead time to diagnosis was negatively associated with DQ at all-time points, but this result did not reach statistical significance (Table 4).

\section{Long-term Developmental Trends in Children with MDs}

Long-term follow-up over the course of 7.70 years (from pre-first to post-sixth) showed declining trends over all studied periods (Figure 1). During the lead time to diagnosis, the slope of DQ 
TABLE 3 | Developmental quotient (DQ) for subgroups of children with mitochondrial disease (total, $n=53$ ).

\begin{tabular}{|c|c|c|c|c|c|c|c|c|c|c|c|c|c|}
\hline \multirow[t]{2}{*}{$\mathbf{D Q}$} & & \multicolumn{3}{|c|}{ Syndromic diagnosis } & \multicolumn{3}{|c|}{ Drug-resistant epilepsy } & \multicolumn{3}{|c|}{ Diffuse brain atrophy } & \multicolumn{3}{|c|}{ Clinical rating } \\
\hline & & Leigh disease & Non-specific & $p$ & No & Yes & $p$ & No & Yes & $p$ & Improving-static & Deteriorating & $p$ \\
\hline \multirow{4}{*}{$\begin{array}{l}\text { Pre- } \\
\text { diagnostic } \\
\text { evaluation }\end{array}$} & Pre-first & $\begin{array}{c}68.3 \pm 35.4 \\
(n=4)\end{array}$ & $\begin{array}{l}52.5 \pm 33.4 \\
\quad(n=14)\end{array}$ & 0.425 & $\begin{array}{c}61.5 \pm 30.4 \\
(n=7)\end{array}$ & $\begin{array}{c}52.6 \pm 36.3 \\
\quad(n=11)\end{array}$ & 0.536 & $\begin{array}{c}78.6 \pm 20.0 \\
(n=6)\end{array}$ & $\begin{array}{c}44.3 \pm 35.3 \\
(n=11)\end{array}$ & $0.047^{\star}$ & $\begin{array}{c}66.9 \pm 31.8 \\
(n=9)\end{array}$ & $\begin{array}{c}45.2 \pm 33.2 \\
(n=9)\end{array}$ & 0.177 \\
\hline & $\begin{array}{l}\text { Difference Pre-first } \\
\text { vs Pre-second }\end{array}$ & $\begin{array}{c}23.6 \pm 38.6 \\
(n=4)\end{array}$ & $\begin{array}{c}12.2 \pm 20.4 \\
(n=14)\end{array}$ & 0.433 & $\begin{array}{c}27.2 \pm 22.2 \\
(n=7)\end{array}$ & $\begin{array}{c}6.8 \pm 23.6 \\
(n=11)\end{array}$ & 0.151 & $\begin{array}{c}17.0 \pm 21.9 \\
(n=6)\end{array}$ & $\begin{array}{c}14.3 \pm 27.9 \\
(n=11)\end{array}$ & 0.841 & $\begin{array}{c}7.1 \pm 9.24 \\
(n=9)\end{array}$ & $\begin{array}{c}22.4 \pm 32.7 \\
(n=9)\end{array}$ & 0.207 \\
\hline & Pre-second & $\begin{array}{l}55.8 \pm 25.9 \\
\quad(n=11)\end{array}$ & $\begin{array}{c}49.1 \pm 33.7 \\
(n=35)\end{array}$ & 0.545 & $\begin{array}{c}54.2 \pm 31.0 \\
(n=23)\end{array}$ & $\begin{array}{c}48.6 \pm 33.1 \\
(n=24)\end{array}$ & 0.632 & $\begin{array}{c}63.9 \pm 33.2 \\
(n=16)\end{array}$ & $\begin{array}{c}44.8 \pm 30.1 \\
(n=30)\end{array}$ & 0.055 & $\begin{array}{l}68.5 \pm 27.1 \\
\quad(n=19)\end{array}$ & $\begin{array}{c}39.7 \pm 29.9 \\
(n=28)\end{array}$ & $0.002^{\star *}$ \\
\hline & $\begin{array}{l}\text { Difference Pre-second } \\
\text { vs diagnosis }\end{array}$ & $\begin{aligned} 20.7 & \pm 16.2 \\
(n & =8)\end{aligned}$ & $\begin{array}{c}16.7 \pm 24.3 \\
(n=24)\end{array}$ & 0.676 & $\begin{array}{c}22.3 \pm 18.4 \\
(n=13)\end{array}$ & $\begin{array}{c}16.4 \pm 25.3 \\
(n=20)\end{array}$ & 0.250 & $\begin{aligned} 20.1 & \pm 24.8 \\
(n & =9)\end{aligned}$ & $\begin{array}{c}18.6 \pm 22.8 \\
(n=23)\end{array}$ & 0.878 & $\begin{array}{c}18.4 \pm 18.0 \\
(n=13)\end{array}$ & $\begin{array}{c}18.9 \pm 25.7 \\
(n=20)\end{array}$ & 0.951 \\
\hline $\begin{array}{l}\text { Diagnostic } \\
\text { evaluation }\end{array}$ & Diagnosis & $\begin{array}{c}31.3 \pm 26.3 \\
(n=8)\end{array}$ & $\begin{array}{c}32.5 \pm 25.2 \\
(n=29)\end{array}$ & 0.903 & $\begin{array}{c}36.0 \pm 23.6 \\
(n=17)\end{array}$ & $\begin{array}{c}30.4 \pm 25.7 \\
(n=22)\end{array}$ & 0.392 & $\begin{array}{c}43.1 \pm 19.3 \\
(n=14)\end{array}$ & $\begin{array}{c}26.7 \pm 26.3 \\
(n=24)\end{array}$ & 0.051 & $\begin{array}{c}44.5 \pm 22.8 \\
(n=15)\end{array}$ & $\begin{array}{c}25.6 \pm 23.3 \\
(n=24)\end{array}$ & $0.018^{\star}$ \\
\hline \multirow{4}{*}{$\begin{array}{l}\text { Post- } \\
\text { diagnostic } \\
\text { evaluation }\end{array}$} & $\begin{array}{l}\text { Difference Diagnosis } \\
\text { vs post-first }\end{array}$ & $\begin{array}{c}11.8 \pm 7.5 \\
(n=5)\end{array}$ & $\begin{array}{c}5.4 \pm 8.2 \\
(n=14)\end{array}$ & 0.147 & $\begin{array}{c}8.8 \pm 9.1 \\
(n=8)\end{array}$ & $\begin{array}{c}4.1 \pm 10.0 \\
(n=13)\end{array}$ & 0.595 & $\begin{array}{l}5.4 \pm 15.9 \\
(n=5)\end{array}$ & $\begin{array}{c}5.7 \pm 7.9 \\
(n=15)\end{array}$ & 0.952 & $\begin{array}{c}8.7 \pm 9.9 \\
(n=10)\end{array}$ & $\begin{array}{c}3.3 \pm 9.4 \\
(n=11)\end{array}$ & 0.214 \\
\hline & Post-first & $\begin{array}{c}30.2 \pm 21.3 \\
(n=5)\end{array}$ & $\begin{array}{c}25.4 \pm 25.3 \\
(n=14)\end{array}$ & 0.709 & $\begin{array}{c}27.4 \pm 24.7 \\
(n=8)\end{array}$ & $\begin{array}{c}29.6 \pm 25.8 \\
(n=13)\end{array}$ & 0.697 & $\begin{array}{c}44.0 \pm 23.0 \\
(n=5)\end{array}$ & $\begin{array}{c}23.9 \pm 24.9 \\
(n=15)\end{array}$ & 0.130 & $\begin{array}{c}38.0 \pm 22.6 \\
(n=10)\end{array}$ & $\begin{array}{c}20.4 \pm 24.6 \\
(n=11)\end{array}$ & 0.106 \\
\hline & $\begin{array}{l}\text { Difference Post-first } \\
\text { vs post-second }\end{array}$ & $\begin{array}{l}5.5 \pm 5.0 \\
(n=4)\end{array}$ & $\begin{array}{c}11.3 \pm 18.6 \\
(n=10)\end{array}$ & 0.559 & $\begin{array}{c}2.8 \pm 4.2 \\
(n=4)\end{array}$ & $\begin{array}{c}12.1 \pm 17.3 \\
(n=11)\end{array}$ & 0.343 & $\begin{array}{c}6.0 \pm 6.7 \\
(n=4)\end{array}$ & $\begin{array}{c}12.4 \pm 17.9 \\
(n=10)\end{array}$ & 0.507 & $\begin{array}{c}16.8 \pm 23.2 \\
(n=6)\end{array}$ & $\begin{array}{c}4.9 \pm 3.6 \\
(n=9)\end{array}$ & 0.267 \\
\hline & Post-second & $\begin{array}{c}17.0 \pm 18.9 \\
(n=4)\end{array}$ & $\begin{array}{c}13.3 \pm 13.7 \\
(n=10)\end{array}$ & 0.687 & $\begin{array}{c}13.6 \pm 20.0 \\
(n=4)\end{array}$ & $\begin{array}{c}19.1 \pm 19.2 \\
(n=11)\end{array}$ & 0.343 & $\begin{array}{c}33.8 \pm 10.0 \\
(n=4)\end{array}$ & $\begin{array}{c}23.7 \pm 13.4 \\
(n=10)\end{array}$ & $0.033^{*}$ & $\begin{array}{c}25.33 \pm 15.9 \\
(n=6)\end{array}$ & $\begin{array}{c}12.43 \pm 19.8 \\
(n=9)\end{array}$ & 0.106 \\
\hline
\end{tabular}

Data given as total number or mean $\pm S D$. Non-parametric Mann-Whitney analysis with Bonferroni correction: ${ }^{*} p<0.05,{ }^{* *} p<0.01$. Pre-first, first pre-diagnostic evaluation; pre-second, second pre-diagnostic evaluation; post-first, first post-diagnostic evaluation; post-second, second post-diagnostic evaluation. 
declined significantly, reflecting a faster deterioration compared with that noted between diagnosis and post-first. The DQ declined steeply also between post-first and post-third. These declining patterns were comparable for the overall development,

TABLE 4 | Correlation between the developmental quotient (DQ) and age at symptom onset, age at diagnosis, and lead time to diagnosis.

\begin{tabular}{llccc}
\hline DQ & & $\begin{array}{c}\text { Age at } \\
\text { symptom } \\
\text { onset }\end{array}$ & $\begin{array}{c}\text { Age at } \\
\text { diagnosis }\end{array}$ & $\begin{array}{c}\text { Lead time to } \\
\text { diagnosis }\end{array}$ \\
\hline $\begin{array}{l}\text { Pre-diagnostic } \\
\text { evaluation }\end{array}$ & Pre-first & 0.048 & -0.283 & -0.305 \\
$\begin{array}{l}\text { Diagnostic } \\
\text { evaluation }\end{array}$ & Diagnosis & 0.247 & -0.086 & -0.266 \\
$\begin{array}{l}\text { Post-diagnostic } \\
\text { evaluation }\end{array}$ & Post-first & $0.438^{*}$ & 0.225 & -0.141 \\
\hline
\end{tabular}

Significant correlation: ${ }^{*} p<0.05$.

Pre-first, first pre-diagnostic evaluation; pre-second, second pre-diagnostic evaluation; post-first, first post-diagnostic evaluation; post-second, second post-diagnostic evaluation. as well as for the five development subdomains describing gross motor, fine motor, social-personal, language, and cognitiveadaptive skills.

The long-term follow-up over the course of 5.45 years (from prefirst to post-second) showed consistent patterns of developmental deterioration (Table 5). Significant developmental deteriorations were noted for the overall DQ between pre-first and pre-second (difference, $14.7 \pm 24.6, p=0.021$ ), as well as between pre-second and diagnosis (difference, $18.7 \pm 22.7, p<0.001$ ). Similar patterns of developmental deteriorations were observed in terms of gross motor (difference: pre-first to pre-second, $10.7 \pm 20.6, p=0.042$; pre-second to diagnosis, $13.7 \pm 25.4, p=0.004)$ and fine motor skills (difference: pre-first to pre-second, $12.6 \pm 20.7, p=0.020$; pre-second to diagnosis, $17.9 \pm 26.3, p<0.001)$. In addition, evident developmental deteriorations were observed in terms of social-personal (difference, $20.2 \pm 31.6, p<0.001$ ), language (difference, $21.5 \pm 27.8, p<0.001$ ), and cognitive-adaptive skills (difference, $20.7 \pm 27.9, p<0.001$ ), but only between pre-second and diagnosis. In contrast, no significant differences were found for the post-diagnostic evaluations (i.e., between diagnosis and post-first, and between post-first and post-second).

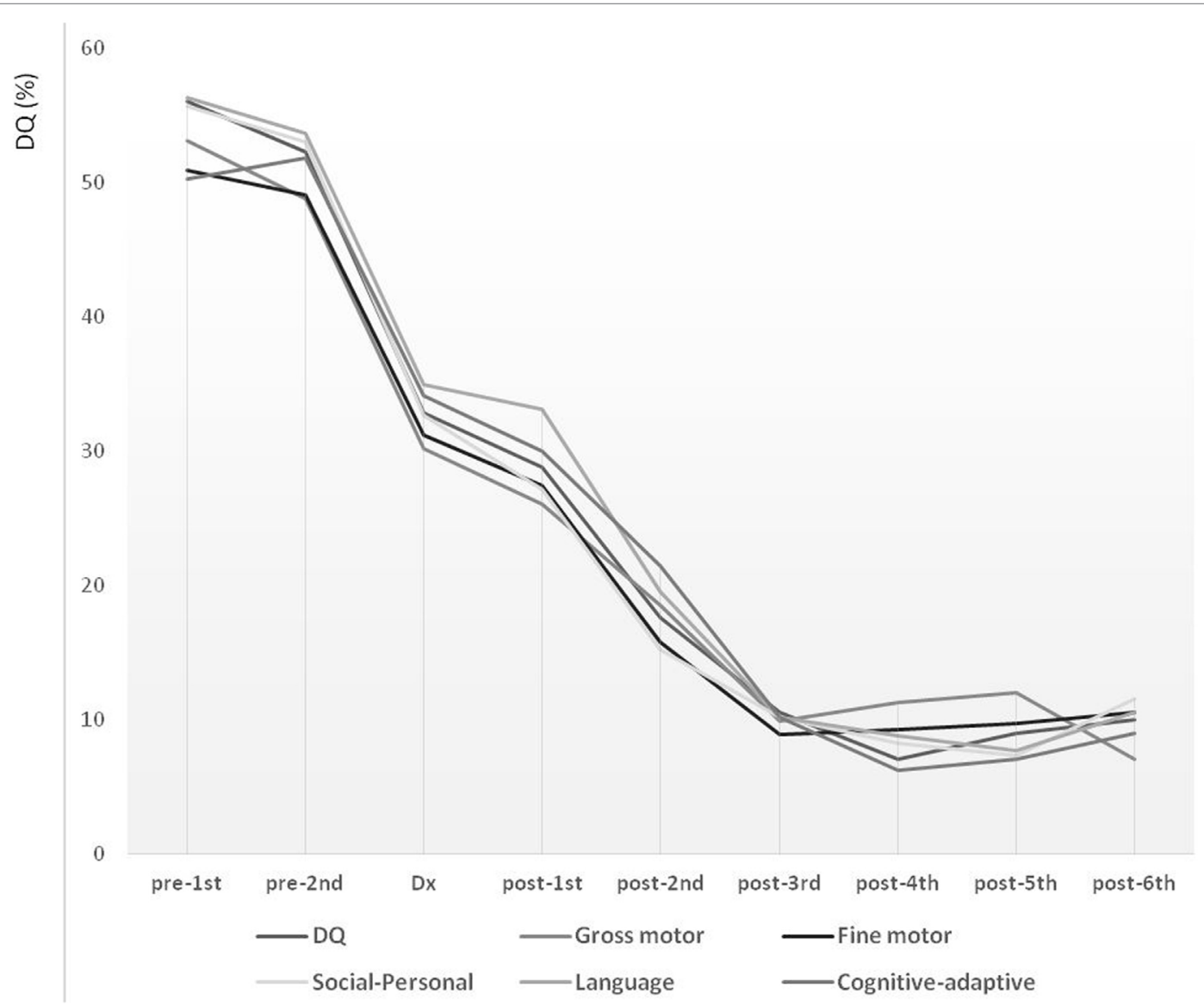

FIGURE 1 | Long-term developmental trends in children with mitochondrial disease evaluated at nine time points over the course of 7.7 years Development level is expressed in terms of developmental quotient (DQ). Pre-diagnostic developmental evaluations were performed twice (pre-first and pre-second), followed by a one-time diagnostic evaluation (Dx) and six post-diagnostic developmental evaluations (post-first, post-second, post-third, post-fourth, post-fifth, and post-sixth). 
TABLE 5 | Evolution of DQ from the first pre-diagnostic evaluation to the second post-diagnostic evaluation, in terms of various skills of children with mitochondrial diseases (total, $n=53$ ).

\begin{tabular}{|c|c|c|c|c|c|c|}
\hline DQ & Subtrahend vs minuend & $n$ & Subtrahend & Minuend & Difference & $p$ \\
\hline \multirow[t]{4}{*}{ Total } & Pre-first vs pre-second & 18 & $56.0 \pm 33.5$ & $41.3 \pm 29.4$ & $14.7 \pm 24.6$ & $0.021^{\star}$ \\
\hline & Pre-second vs diagnosis & 33 & $48.8 \pm 31.3$ & $30.1 \pm 25.0$ & $18.7 \pm 22.7$ & $<0.001^{\star \star \star}$ \\
\hline & Diagnosis vs post-first & 21 & $34.6 \pm 26.2$ & $28.8 \pm 24.8$ & $5.9 \pm 9.8$ & 0.013 \\
\hline & Post-first vs post-second & 15 & $27.3 \pm 25.5$ & $17.6 \pm 18.9$ & $9.7 \pm 15.3$ & 0.029 \\
\hline \multirow[t]{4}{*}{ Gross motor } & Pre-first vs pre-second & 18 & $53.1 \pm 32.9$ & $42.4 \pm 31.4$ & $10.7 \pm 20.6$ & $0.042^{*}$ \\
\hline & Pre-second vs diagnosis & 33 & $42.4 \pm 32.8$ & $28.7 \pm 23.4$ & $13.7 \pm 25.4$ & $0.004^{\star \star}$ \\
\hline & Diagnosis vs post-first & 21 & $30.1 \pm 22.1$ & $26.0 \pm 24.3$ & $4.1 \pm 14.7$ & 0.223 \\
\hline & Post-first vs post-second & 15 & $25.7 \pm 24.8$ & $18.5 \pm 18.2$ & $7.1 \pm 12.9$ & 0.051 \\
\hline \multirow[t]{4}{*}{ Fine motor } & Pre-first vs pre-second & 18 & $50.9 \pm 35.8$ & $38.3 \pm 27.6$ & $12.6 \pm 20.7$ & $0.020^{\star}$ \\
\hline & Pre-second vs diagnosis & 33 & $45.1 \pm 34.1$ & $27.2 \pm 28.3$ & $17.9 \pm 26.3$ & $<0.001^{\star \star \star}$ \\
\hline & Diagnosis vs post-first & 21 & $32.7 \pm 33.1$ & $27.4 \pm 28.0$ & $5.2 \pm 18.6$ & 0.209 \\
\hline & Post-first vs post-second & 15 & $25.3 \pm 27.8$ & $15.7 \pm 19.9$ & $9.5 \pm 18.1$ & 0.062 \\
\hline \multirow[t]{4}{*}{ Social-personal } & Pre-first vs pre-second & 18 & $55.7 \pm 39.1$ & $40.2 \pm 35.0$ & $15.5 \pm 31.6$ & 0.053 \\
\hline & Pre-second vs diagnosis & 33 & $51.9 \pm 35.9$ & $31.6 \pm 27.7$ & $20.2 \pm 31.6$ & $<0.001^{\star \star \star}$ \\
\hline & Diagnosis vs post-first & 21 & $34.9 \pm 30.0$ & $27.2 \pm 24.3$ & $7.7 \pm 21.1$ & 0.110 \\
\hline & Post-first vs post-second & 15 & $25.4 \pm 23.6$ & $15.2 \pm 14.5$ & $10.1 \pm 21.6$ & 0.104 \\
\hline \multirow[t]{4}{*}{ Language } & Pre-first vs pre-second & 18 & $56.4 \pm 37.8$ & $45.4 \pm 31.7$ & $10.9 \pm 22.7$ & 0.057 \\
\hline & Pre-second vs diagnosis & 33 & $52.6 \pm 33.9$ & $31.0 \pm 27.6$ & $21.5 \pm 27.8$ & $<0.001^{\star \star \star}$ \\
\hline & Diagnosis vs post-first & 21 & $37.8 \pm 30.2$ & $33.1 \pm 29.4$ & $4.6 \pm 10.7$ & 0.064 \\
\hline & Post-first vs post-second & 15 & $32.8 \pm 31.8$ & $19.6 \pm 23.4$ & $13.2 \pm 18.6$ & 0.020 \\
\hline \multirow[t]{4}{*}{ Cognitive-adaptive } & Pre-first vs pre-second & 18 & $50.3 \pm 39.3$ & $38.6 \pm 35.3$ & $11.7 \pm 31.3$ & 0.131 \\
\hline & Pre-second vs diagnosis & 33 & $51.6 \pm 37.1$ & $30.8 \pm 29.0$ & $20.7 \pm 27.9$ & $<0.001^{\star \star \star}$ \\
\hline & Diagnosis vs post-first & 21 & $35.8 \pm 33.1$ & $30.0 \pm 29.2$ & $5.8 \pm 14.5$ & 0.081 \\
\hline & Post-first vs post-second & 15 & $30.7 \pm 31.1$ & $21.4 \pm 24.6$ & $9.2 \pm 16.1$ & 0.051 \\
\hline
\end{tabular}

Data given as total number or mean $\pm S D$. Differences assessed via the paired $t$-test with Bonferroni correction: ${ }^{*} p<0.05,{ }^{* *} p<0.01,{ }^{* * *} p<0.001$. Pre-first, first pre-Dx; pre-second, second pre-Dx; post-first, first post-diagnostic state; post-second, second post-diagnostic state; DQ, developmental quotient; Dx, diagnostic evaluation.

\section{Long-term Development Before and After Diagnosis}

Repeated measures ANOVA revealed a significant difference in the rate of developmental decline for the pre- and post-diagnosis periods $(p<0.001)$. The subsequent post hoc test indicated that these differences were significant for all types of skills.

Repeated measures analysis showed a persistent pattern of developmental deterioration between the pre-diagnostic and post-diagnostic periods for overall development [difference, 16.1; $95 \%$ confidence interval $(\mathrm{CI}), 6.3-25.8, p=0.001]$, as well as gross motor (difference, 12.8 ; 95\% CI, 1.8-23.8, $p=0.020$ ), fine motor (difference, 16.2; 95\% CI, 2.2-30.2, $p=0.020$ ), social-personal (difference, 16.3 ; 95\% CI, 0.8-31.8, $p=0.037$ ), language (difference, 20.7; 95\% CI, 6.1-35.2, $p=0.005$ ), and cognitive-adaptive (difference, 15.3; 95\% CI, 1.0-29.5, $p=0.033$ ) skills (Table 6). The overall DQ showed a decline from the pre-diagnostic through the post-diagnostic periods, suggesting an overall deterioration.

\section{DISCUSSION}

In the present study, the global developmental trends were evaluated over a follow-up period of 7.70 years at nine time points and showed a decline over all periods. However, even with consistently declining patterns, the characteristics of developmental deterioration were disparate. The overall developmental decline
TABLE 6 | Repeated measures ANOVA for long-term development of various skills in children with mitochondrial diseases $(n=18)$.

\begin{tabular}{|c|c|c|c|c|}
\hline DQ & Difference & $p$ & $\begin{array}{c}\text { Difference (95\% } \\
\text { confidence interval) }\end{array}$ & Post hoc \\
\hline \multirow[t]{3}{*}{ Total } & & $<0.001$ & & \\
\hline & Pre-Dx & & $16.1(6.3-25.8)$ & $0.001^{\star \star}$ \\
\hline & Dx-post & & $7.7(2.4-13.0)$ & $0.004^{\star \star}$ \\
\hline \multirow[t]{3}{*}{ Gross motor } & & $<0.001$ & & \\
\hline & Pre-Dx & & $12.8(1.8-23.8)$ & $0.020^{\star}$ \\
\hline & Dx-post & & $6.8(0.6-14.3)$ & 0.080 \\
\hline \multirow[t]{3}{*}{ Fine motor } & & $<0.001$ & & \\
\hline & Pre-Dx & & $16.2(2.2-30.2)$ & $0.020^{\star}$ \\
\hline & Dx-post & & $7.7(1.0-16.4)$ & 0.095 \\
\hline \multirow{3}{*}{$\begin{array}{l}\text { Social- } \\
\text { personal }\end{array}$} & & $<0.001$ & & \\
\hline & Pre-Dx & & $16.3(0.8-31.8)$ & $0.037^{*}$ \\
\hline & Dx-post & & $11.4(0.7-23.5)$ & 0.071 \\
\hline \multirow[t]{3}{*}{ Language } & & $<0.001$ & & \\
\hline & Pre-Dx & & 20.7 (6.1-35.2) & $0.005^{\star \star}$ \\
\hline & Dx-post & & $4.3(2.8-11.5)$ & 0.381 \\
\hline \multirow{3}{*}{$\begin{array}{l}\text { Cognitive- } \\
\text { adaptive }\end{array}$} & & 0.001 & & \\
\hline & Pre-Dx & & $15.3(1.0-29.5)$ & $0.033^{\star}$ \\
\hline & Dx-post & & $6.4(2.7-15.6)$ & 0.240 \\
\hline
\end{tabular}

General linear model, repeated measures with Bonferroni adjustment: * $p<0.05$, ${ }^{* *} p<0.01$. DQ, developmental quotient; pre, pre-diagnostic evaluation; post, postdiagnostic evaluation; Dx, diagnostic evaluation. 
pattern could be classified into five phases: (1) pre-diagnostic initial decline phase; (2) pre-diagnostic accelerated decline phase; (3) post-diagnostic alleviated phase; (4) post-diagnostic reaccelerated decline phase; and (5) post-diagnostic stagnant phase.

Considering the pre-diagnostic phase as lead time, the DQ declined continuously, with the initial decline starting from the first onset of symptoms, followed by an accelerated deteriorating phase until diagnosis. During the initial period from diagnosis to the first post-diagnostic evaluation ( $\sim 1$ year), an impeded phase of developmental decline was noted, which appeared alleviated compared with the decline noted in previous phases. This was followed by an accelerated period of decline for $\sim 2$ years. Finally, $\sim 3$ years after the diagnosis, the decline reached a stagnant phase. In this phase, the developmental deterioration appeared to be reaching the lowest and terminal level of $10 \%$ of the DQ. These declining patterns were similar for the overall development and all types of skills, including the gross motor, fine motor, socialpersonal, language, and cognitive-adaptive. However, a more detailed analysis revealed that the delayed development in gross and fine motor skills exhibited more significant deterioration even in the pre-diagnostic initial decline phase.

In terms of the lead time, the developmental decline accelerated from the first onset of symptoms to the time of confirmation of diagnosis. In contrast, right after the diagnosis, a post-diagnostic alleviated phase was manifested. These facets of the developmental course highlight the importance of shortening the lead time as well as of early detection of the first significant symptom. A shorter lead time is important in achieving a longer developmentally alleviated phase or a prolonged period before reaching the stagnant phase and the lowest and terminal level of development.

As developmental delay is one of the first common symptoms in children with MD (16), vigilance and careful observation of any motor delay in children are integral to early diagnosis. With more structural attention and information, motor delays might be easier to detect by caregivers because of their comparatively evident developmental milestones in earlier childhood compared with language, cognitive, or social development (7). In addition, any motor delay is usually enough for a child to be referred to professionals, and additional signs of any developmental delays in the language, social, and cognitive domains should be further evaluated for etiological diagnosis. Hence, early detection of motor delay and other developmental signs of delay is important in children with MD to shorten the lead time.

Furthermore, the effects of diagnosis and intervention should not be overlooked in children with MD. Such a standardized screening tool to detect MDs early at patients' routine visits to the pediatricians would be ideal. However, making the correct diagnosis is exceedingly problematic because of the heterogeneous features of this disorder $(17,18)$. MD may present at any age, with a spectrum of symptoms and signs, to several medical specialties. And there are no standard guidelines for the investigation of $\operatorname{MD}(2,3)$. Diagnostic difficulty results not only from the wide spectrum of symptoms and signs that an individual patient may have but also from the absence of a reliable screening or diagnostic biomarker that is both sensitive and specific in all cases of MD (4). Discussion with a clinician who has expertise in MD is advised in order to guide investigation, in particular with respect to laboratory facilities and handling of specimens (2). Therefore, it might not be easy to adopt a standardized screening tool to detect them early in MD.

Confirming the diagnosis of MD is important to both the parents and physician, because confirmation of diagnosis could relieve the parents' anxiety and helplessness caused by the uncertainty of an undiagnosed condition and encourage them to seek various therapies for their children. For physicians, confirming the diagnosis of $\mathrm{MD}$ is fundamental in planning therapeutic intervention $(17,18)$.

The appearance of a post-diagnostic alleviated phase in this study is notable and possibly even inspiring to physicians who have only been using symptomatic or supportive treatment for MD. The possibility of a positive effect of the treatment on patients' development, including mitochondrial cocktails, such as coenzyme Q, carnitine, and various vitamins, which were provided to the children with MD in this study, should not be dismissed. Notably, the children with $\mathrm{MD}$ in this study had been taking a mitochondrial cocktail immediately after receiving a confirmed diagnosis. Accordingly, the necessity of an early diagnosis, consecutive symptomatic treatment, general supportive care, and early administration of possible medicine to shorten the lead time to diagnosis and alleviate the patients' developmental regression is suggested.

We found that diffuse brain atrophy, the clinical rating provided by the physician, and the age at first symptom onset significantly affected the developmental level and decline. However, no significant effect was noted for syndromic diagnosis and epilepsy severity, suggesting that these aspects may not directly reflect the developmental condition of the patients. Because of the heterogeneous symptoms of MDs (14), syndromic diagnosis based on a combination of particular symptoms might not be strongly associated with the developmental function of children with MDs. Additionally, epilepsy-related factors are not the main factors that influence developmental function in such patients, but are nonetheless important manifestations of MDs (14). Instead, diffuse brain atrophy might largely account for the developmental level as a measure of brain function with structural change $(16,19)$. In particular, all pediatric patients included in the present study presented with central nervous system involvement. In addition, more than half had diffuse brain atrophy, which was associated with lower developmental level at all-time points and over all periods considered, from the early stage of the pre-diagnostic period through the lowest, stagnant, and terminal phase. The concept of clinical ratings (20) provided by the treating physician could be regarded as a good predictor of longterm developmental prognosis because it reflects the characteristics of the patient's general state and multiorgan involvement.

This study has several limitations. This is a preliminary study with a small number of patients, and the overall cohort was not followed up consistently since we reviewed the data retrospectively. Moreover, in spite of the advantage of other developmental scales such as Bayley scale of Infants Development, KICDT was used as the most frequently and repeatedly used measure in outpatient or inpatient clinical settings in the current study. It still has a strength as a possible long-term and repeatedly useful screening tool, however, other developmental measures might be able to give more reliable information about the patients' developmental function. In addition, patients' anthropometric values were not 
included in the current study due to the small number of patients as a preliminary study. Despite such limitations, the present study addresses the lack of data regarding the long-term developmental trajectory and clinical course of pediatric patients with MDs, as such diseases are characterized by diverse and heterogeneous feature manifestations $(7,20,21)$. Even a rough estimate of the long-term developmental trajectory of children with MD could provide important information for diagnosis and development of possible intervention plans. In future study, we hope that we could overcome this limitation on the heterogeneity of MD with a larger number of patients and include prospective data as well based on the understanding of developmental course of pediatric MD from current preliminary data.

Despite the study limitations, our findings highlight the importance of DQ in the prediction and rating of the long-term functional course of children with MD. There has been no other established predictor in terms of patient's functional measure in pediatric MD because of its difficulties of expecting the clinical course. In the perspective of this limitation, development quotient might be a candidate as a predictor or a measure in pediatric MD by giving the functional level of patients, which is a very meaningful value in pediatrics and could be obtained even from pediatric MD patients with much delayed developmental level. Our observations suggest that, upon confirmation of the diagnosis by the physician, measures, such as symptomatic treatment, general supportive care, and early administration of medicine, are encouraged to shorten the lead time to diagnosis and thereby minimize the patients' developmental regression.

\section{REFERENCES}

1. Noorda G, Hermans-Peters M, Smeitink J, van Achterberg T, Kemps H, Goverde W, et al. Mitochondrial disease: needs and problems of children, their parents and family. A systematic review and pilot study into the need for information of parents during the diagnostic phase. J Inherit Metab Dis (2007) 30:333-40. doi:10.1007/s10545-007-0426-0

2. Kisler JE, Whittaker RG, McFarland R. Mitochondrial diseases in childhood: a clinical approach to investigation and management. Dev Med Child Neurol (2010) 52(5):422-33. doi:10.1111/j.1469-8749.2009.03605.x

3. Horvarth R, Hudson G, Ferrari G, Fütterer N, Ahola S, Lamantea E, et al. Phenotypic spectrum associated with mutations of the polymerase gamma gene. Brain (2006) 129:1637-9. doi:10.1093/brain/awl088

4. Mitochondrial Medicine Society's Committee on Diagnosis, Haas RH, Parikh S, Falk MJ, Saneto RP, Wolf NI, et al. The in depth evaluation of suspected mitochondrial disease. Mol Genet Metab (2008) 94:16-37. doi:10.1016/j. ymgme.2007.11.018

5. Koene S, Wortmann SB, de Vries MC, Jonckheere AI, Morava E, de Groot IJ, et al. Developing outcome measures for pediatric mitochondrial disorders: which complaints and limitations are most burdensome to patients and their parents? Mitochondrion (2013) 13:15-24. doi:10.1016/j.mito.2012.11.002

6. Alvero AM, Austin J. The effects of conducting behavioral observations on the behavior of the observer. J Appl Behav Anal (2004) 37:457-68. doi:10.1901/ jaba.2004.37-457

7. Debray FG, Lambert M, Chevalier I, Robitaille Y, Decarie JC, Shoubridge EA, et al. Long-term outcome and clinical spectrum of 73 pediatric patients with mitochondrial diseases. Pediatrics (2007) 119:722-33. doi:10.1542/ peds.2006-1866

8. Msall ME, Tremont MR. Measuring functional status in children with genetic impairments. Am JMed Genet (1999) 89:62-74. doi:10.1002/ (SICI) 1096-8628(19990625)89:2<62::AID-AJMG3>3.0.CO;2-T

\section{ETHICS STATEMENT}

All procedures were approved by the institutional review board of Gangnam Severance Hospital in Seoul, Korea.

\section{AUTHOR CONTRIBUTIONS}

SE conceptualized and designed the study, carried out the data analysis, drafted the initial manuscript, reviewed and revised the manuscript, and approved the final manuscript as submitted. Y-ML conceptualized and designed the study, critically reviewed and revised the manuscript, and approved the final manuscript as submitted. Both listed authors meet the appropriate authorship criteria. No one who qualifies for authorship has been omitted from the list. Contributors and their funding sources have been properly acknowledged, and authors and contributors have approved the acknowledgment of their contributions.

\section{FUNDING}

This research was supported by the Basic Science Research Program through the National Research Foundation of Korea (NRF) funded by the Ministry of Science, ICT and Future Planning (2014-31-0662 and 2015-31-0580), and by a grant of the Korea Health Technology R\&D Project through the Korea Health Industry Development Institute (KHIDI), funded by the Ministry of Health and Welfare, Republic of Korea (HI16C0673).

9. Bernier FP, Boneh A, Dennett X, Chow CW, Cleary MA, Thorburn DR. Diagnostic criteria for respiratory chain disorders in adults and children. Neurology (2002) 59:1406-11. doi:10.1212/01.WNL.0000033795.17156.00

10. Yatsuga S, Povalko N, Nishioka J, Katayama K, Kakimoto N, Matsuishi T, et al. MELAS: a nationwide prospective cohort study of 96 patients in Japan. Biochim Biophys Acta (2012) 1820:619-24. doi:10.1016/j.bbagen.2011.03.015

11. Rahman S, Blok RB, Dahl HH, Danks DM, Kirby DM, Chow CW, et al. Leigh syndrome: clinical features and biochemical and DNA abnormalities. Ann Neurol (1996) 39:343-51. doi:10.1002/ana.410390311

12. Kwan P, Arzimanoglou A, Berg AT, Brodie MJ, Allen Hauser W, Mathern G, et al. Definition of drug resistant epilepsy: consensus proposal by the ad hoc task force of the ILAE commission on therapeutic strategies. Epilepsia (2010) 51:1069-77. doi:10.1111/j.1528-1167.2009.02397.x

13. Skladal D, Sudmeier C, Konstantopoulou V, Stöckler-Ipsiroglu S, PleckoStartinig B, Bernert G, et al. The clinical spectrum of mitochondrial disease in 75 pediatric patients. Clin Pediatr (Phila) (2003) 42:703-10. doi:10.1177/000992280304200806

14. Finsterer J. Mitochondriopathies. Eur J Neurol (2004) 11:163-86. doi:10.1046/j.1351-5101.2003.00728.x

15. Kim HJ, Shin JI. A study of the correlation between BSID-III and KICDT for children with developmental delay. J Phys Ther Sci (2015) 27:269-71. doi:10.1589/jpts.27.269

16. Kim JT, Lee YJ, Lee YM, Kang HC, Lee JS, Kim HD. Clinical characteristics of patients with non-specific and non-categorized mitochondrial diseases. Acta Paediatr (2009) 98:1825-9. doi:10.1111/j.1651-2227.2009.01428.x

17. Finsterer J. Central nervous system imaging in mitochondrial disorders. Can J Neurol Sci (2009) 36:143-53. doi:10.1017/S0317167100006508

18. Finsterer J. Laminar cortical necrosis in mitochondrial disorders. Clin Neurol Neurosurg (2009) 111:655-8. doi:10.1016/j.clineuro.2009.07.010

19. Finsterer J. Central nervous system manifestations of mitochondrial disorders. Acta Neurol Scand (2006) 114:217-38. doi:10.1111/j.1600-0404.2006.00671.x 
20. Scaglia F, Towbin JA, Craigen WJ, Belmont JW, Smith EO, Neish SR, et al. Clinical spectrum, morbidity, and mortality in 113 pediatric patients with mitochondrial disease. Pediatrics (2004) 114:925-31. doi:10.1542/peds.2004-0718

21. Finsterer J, Harbo HF, Baets J, Van Broeckhoven C, Di Donato S, Fontaine B, et al. EFNS guidelines on the molecular diagnosis of mitochondrial disorders. Eur J Neurol (2009) 16:1255-64. doi:10.1111/j.1468-1331.2009.02811.x

Conflict of Interest Statement: The authors declare that they have no financial relationships relevant to this article. The funders had no role in study design, data collection, data analysis, interpretation of results, manuscript preparation, or decision to submit the article for publication.

Copyright (C) 2017 Eom and Lee. This is an open-access article distributed under the terms of the Creative Commons Attribution License (CC BY). The use, distribution or reproduction in other forums is permitted, provided the original author(s) or licensor are credited and that the original publication in this journal is cited, in accordance with accepted academic practice. No use, distribution or reproduction is permitted which does not comply with these terms 